\title{
Effect of Apical Root Canal Perforation Size on Push-out Bond Strength of Glass Fiber Dowels
}

\author{
Abdulelah M BinMahfooz ${ }^{1}$, Mohammed A Sindi ${ }^{2}$, Tala H Alsohaibi ${ }^{3}$, Hala H Jabbad ${ }^{4}$, Aiman O Johar ${ }^{5}$
}

\begin{abstract}
Aim: To evaluate in vitro the effect of apical root canal perforation size on push-out bond strength of glass fiber dowels cemented to sound or perforated root canals using two different adhesive systems.

Materials and materials: A total of 120 human-extracted intact upper central incisors were selected. Teeth were sectioned $3 \mathrm{~mm}$ coronal to cement enamel junction, and the remaining root received endodontic root canal therapy. The roots were divided into two experimental groups according to the root condition: either sound $(n=40)$ or apically perforated $(n=80)$. Dowel spaces were prepared for all specimens to a depth of $10 \mathrm{~mm}$. Roots were restored with glass fiber dowels. The experimental group was further subdivided into four subgroups ( $n=20$ ) according to the adhesive system used and apical perforation size: group l, perforated root $2 \mathrm{~mm}$ apically, dowel cemented using total-etch adhesive cement; group II, perforated root $2 \mathrm{~mm}$ apically, dowel cemented using self-etch adhesive cement; group III, perforated root $4 \mathrm{~mm}$ apically, dowel cemented using total-etch adhesive cement; and group IV, perforated root $4 \mathrm{~mm}$ apically, dowel cemented using self-etch adhesive cement. The control group, sound root, was divided into two subgroups: group l, sound root, dowel cemented using total-etch adhesive cement, and group II, sound root, dowel cemented using self-etch or total-etch adhesive cement. Each root was then cut horizontally, and root segments were prepared to be tested. The bond strength between dowel and dentin was measured with universal testing machine using a push-out test. The two-way analysis of variance (ANOVA) was used to analyze the data and post hoc Tukey's test $(\alpha=0.05)$.

Results: Root canal perforation and the type of adhesive system used resulted in significant differences in push-out bond strength ( $p<0.05)$. Regardless of root canal perforation size, glass fiber dowels in normal root canals had significantly higher mean bond strength values ( $9.2 \pm 1.4$ $\mathrm{MPa}$ ) compared with perforated root canals $(6.1 \pm 1.4 \mathrm{MPa})$. Also, self-etch protocol had significantly higher mean bond strength values $(9.1 \pm$ $1.3 \mathrm{MPa})$ compared with total-etch protocol $(6.2 \pm 2.1 \mathrm{MPa})$.

Conclusion:The apical root perforation size caused a direct effect on the bond strength of the glass fiber dowels cemented to dentin by reducing the bond strength values to the root dentin regardless of the adhesive system used.

Clinical significance: Prior to perforation repair, dentist or endodontist should evaluate the perforation size to predict the treatment outcome. Keywords: Adhesive resin cement, Fiber post, Mineral trioxide aggregate, Root canal treatment.

The Journal of Contemporary Dental Practice (2020): 10.5005/jp-journals-10024-2793
\end{abstract}

\section{INTRODUCTION}

The use of dowel system to restore endodontically treated teeth is a subject that has been studied significantly in dental literature. ${ }^{1,2}$ Removal of pulpal tissue during the root canal therapy leads to dehydration of the treated teeth and subsequent alteration in the physical and chemical property of the teeth. Multiple challenges must be resolved to ensure successful functional, esthetic, and structural rehabilitation of endodontically treated teeth. ${ }^{3}$ Dowels and cores are frequently used in structurally compromised root canal-treated teeth. In such cases, the cementation of a dowel inside the root canal and rebuilding the coronal tooth structure with a core to provide retention for the final restoration is mandatory. ${ }^{4}$ Cast metal posts and cores have been used in clinical scenarios to provide the needed retention and support for the prosthodontic restoration. ${ }^{5}$ The use of prefabricated dowel systems is preferred by the dentists because they offer more appealing esthetic result due to their optical properties, in addition to being more practical, less expensive, and less invasive than cast metal dowel and core systems. ${ }^{6}$

Dental esthetic demand led to an increase in the popularity of dowels, such as fiber and zirconia, to restore endodontically treated teeth. Recently, fiber dowels were considered by the dentists as promising alternatives to cast metal dowels, due to elastic moduli similarity to dentin, producing a favorable stress distribution. ${ }^{7}$

\footnotetext{
1,5Oral and Maxillofacial Prosthodontics Department, King Abdulaziz University, Faculty of Dentistry, Jeddah, Kingdom of Saudi Arabia

${ }^{2-4}$ King Abdulaziz University, Faculty of Dentistry, Jeddah, Saudi Arabia Corresponding Author: Aiman O Johar, Oral and Maxillofacial Prosthodontics Department, King Abdulaziz University, Faculty of Dentistry, Jeddah, Kingdom of Saudi Arabia, Phone: +966 555665565, e-mail: Aojohar@kau.edu.sa
}

How to cite this article: BinMahfooz AM, Sindi MA, Alsohaibi TH, et al. Effect of Apical Root Canal Perforation Size on Push-out Bond Strength of Glass Fiber Dowels. J Contemp Dent Pract 2020;21(5):515-520.

Source of support: Nil

Conflict of interest: None

The multiple advantages of fiber dowels such as corrosion resistance, compatibility, pleasant esthetic outcome, easier removal for nonsurgical re-treatment, and single-visit office placement have increased its popularity among dentists. ${ }^{8}$ Dentists always face a challenge in selecting an appropriate adhesive system to bond dowels to root dentin. Recent improvements in resin cements have added strength to the sealing ability of adhesive resin luting agents. ${ }^{9}$ Resin cements increase the retention and minimize leakage when compared with other cements. ${ }^{10}$ Moreover, multiple types of bonding systems are available to be combined with different luting resins. ${ }^{9,11}$ Failures of endodontic dowels predominantly 
result from either loss of retention ${ }^{12,13}$ or root fracture. Moreover, accidental root perforation as a result of iatrogenic dental care during root canal treatment or post space preparation is considered as a major complication of endodontic and restorative treatments. Such complication leads to loss of integrity of root and adjacent periodontium. ${ }^{14,15}$ Among the various materials used for perforation repair is mineral trioxide aggregate (MTA). Mineral trioxide aggregate is a biocompatible dental cement, which has been recommended to seal perforation at the root level which leads to communication between the tooth and periodontal tissue. It possesses properties that provide optimal sealing and has the ability to induce osteogenesis and cementogenesis. ${ }^{16}$ Although several authors ${ }^{12,13,16}$ stress the importance of bond strength of fiber-reinforced composite (FRC) dowels to root canal dentin, nothing has been reported on the effect of the perforation size on bond strength of the adhesive resin cement to dentin root canal. The purpose of this study was to evaluate the durability of bond strength for glass fiber dowels using two different adhesive systems in sound and apically perforated root canals.

\section{Materials and Methods}

This investigation used one glass fiber dowel system and two adhesive systems in sound and perforated root canals.

\section{Specimens' Selection}

A total of 120 human-extracted intact upper central incisors were selected. The inclusion criteria were fully developed apices and similar teeth size. All the teeth were measured buccolingual and mesiodistal and recorded in millimeters, allowing a maximum deviation of $10 \%$ from the determined mean. The teeth were kept moist by storing them in distilled water with $0.1 \%$ thymol disinfectant at room temperature.

\section{Preparation of Specimens}

All the teeth selected were sectioned by a diamond disk (Brasseler, Savannah, USA) perpendicular to the long axis, $3 \pm 1 \mathrm{~mm}$ coronal to the cementoenamel junction to separate anatomical crown and provide root lengths of $15 \mathrm{~mm}$. Access to the root canals was gained with high-speed rotary diamond cutting bur (Brasseler), and canals were endodontically instrumented. Each canal was widened manually until an ISO size 35 file (Dentsply/Maillefer, Ballaigues, Switzerland) could be inserted to full working length with no root dentin engagement. The pulpal tissues were removed with a barbed broach of suitable size, and root canals were manually instrumented to a working length of $1 \mathrm{~mm}$ coronal to the apical foramen with K-files (Dentsply/Maillefer). The canals were prepared and irrigated with $2 \mathrm{~mL}$ of $15 \%$ ethylenediaminetetraacetic acid (EDTA) irrigating solution between each file size change using \#40 master apical file and after filing is complete. Obturation was performed using a gutta-percha cone (Dentsply/Maillefer) matching the last file size and $\mathrm{AH}-26$ resin sealer (Dentsply/Maillefer). Endodontic spreader (Brasseler) instruments were used to aid in gutta-percha condensation laterally. Modeling wax was used to seal the access cavities and root apexes. After 24 hours, using Gates-Glidden (Dentsply/Maillefer), the gutta-percha was removed from each root canal leaving only $5 \mathrm{~mm}$ at the apex. Such removal led to 10.0 $\mathrm{mm}$ dowels space length. The post space was irrigated with 10 $\mathrm{mL}$ of the EDTA for 20 seconds to wash out any remaining sealer. Furthermore, to completely reach to dry canal surface, sufficient paper points (Dentsply/Maillefer) were used. A dowel space was prepared using the corresponding drill designated for the respective dowel kit (Dentsply/Maillefer). Periodontal probe was fitted inside the root canal post space for measurement verification using an endodontic reference stop and a radiograph. The root canal spaces were prepared using the designated procedure for the respective dowel system.

\section{Grouping of Specimens}

Specimens were divided into two groups, test $(n=80)$ and control ( $n=40)$. Test group was divided into four subgroups according to apical perforation size and the adhesive system used. However, control group was divided to two subgroups according to the adhesive system used only.

\section{Test Groups}

Group I, apically perforated root $2 \mathrm{~mm}$, dowel cemented using total-etch adhesive cement.

Group II, apically perforated root $4 \mathrm{~mm}$, dowel cemented using total-etch adhesive cement.

Group III, apically perforated root $2 \mathrm{~mm}$, dowel cemented using self-etch adhesive cement.

Group IV, apically perforated root $4 \mathrm{~mm}$, dowel cemented using self-etch adhesive cement.

\section{Control Groups}

Group I, sound root, dowel cemented using total-etch adhesive cement.

Group II, sound root, dowel cemented using self-etch adhesive cement.

For root perforation, cavities were prepared on the apical third of each tooth in test groups I, II, III, and IV using a standard diamond rotary instrument (KG Sorensen Indústria e Comércio Ltda., São Paulo, SP, Brazil). Perforation diameters of $2 \mathrm{~mm}$ or $4 \mathrm{~mm}$ were prepared to the gutta-percha depth. One commercial brand of MTA was used according to the manufacturer's recommendations. The pouch of ProRoot MTA (Dentsply/Maillefer) root repair material was opened and the powder was dispensed onto a mixing pad, the end of a ProRoot liquid was pulled off, and a microdose ampoule was squeezed out onto the mixing pad next to the root repair material. The liquid was gradually incorporated into the cement using the ProRoot MTA mixing stick. The material was mixed with the liquid for about 1 minute to hydrate all the powder content in the mix. Using the carrier, the mix was dispensed and condensed into the perforation site using a small plugger (Dentsply/Maillefer). A radiograph was taken to confirm the placement of the ProRoot MTA root repair material. After four hours, ProRoot MTA root repair material was examined for hardening. Dowels in test group I, and group II, were cemented by total-etch adhesive cement (Adper single bond; 3M ESPE, Saint Paul, MN, USA) according to the manufacturer's instructions as well as group I in control. The canal walls were etched with $35 \%$ phosphoric acid for 15 seconds, water rinsed for 10 seconds, and air dried for 3 seconds. Absorbent paper points were then used to sufficiently dry the post space and remove the excess water. Microbrush tips (Microbrush Corp., Grafton, WI, USA) were used to apply two consecutive coats of Adper single bond two adhesive system to the root canal space. The excess of the adhesive was cleaned with an absorbent paper point, and the remaining material was gently air dried and was exposed to light cure using a halogen light source (Woodpecker, LED-B; Gizmo Supply Co., Fountain Valley, CA, USA) for 10 seconds. Dowels in test group III, and group IV, as well as group II in control, were cemented by self-etch adhesive cement (Clearfil SE Bond, Kuraray America, Inc., 
Madison, NY, USA) according to manufacturer's instructions. One drop was dispensed from each of primer and Clearfil bond activator into a well of the dispensing dish and mixed immediately before application. A light-blocking plate was used to avoid exposing the material to an operating light or natural light. The mixture was applied to the entire dowel space with the applicator brush (Microbrush ${ }^{\circledast}$ X, Microbrush Corp.). The entire adherent surface was thoroughly dried with gentle air for 5 seconds. Any excess was removed with a paper point. After wiping away the excess mixture, the adherent surface was dried again and then light cured with a dental curing unit for 10 seconds. Number 2 easy posts were used and cemented with RelyX ${ }^{\mathrm{TM}}$ ultimate adhesive resin cement (Dentsply/Maillefer). Lentulo spiral (Dentsply/Maillefer) \#30 was used to introduce the mixed cement inside the root canal space. Light finger pressure was applied to ensure each dowel is cemented properly into the root canal, and excess luting material was removed. Light activation was performed for 40 seconds.

\section{Thermal Cycling}

The specimens were subjected to thermal cycling for a total of 5,000 cycles between $5^{\circ} \mathrm{C}$ and $50^{\circ} \mathrm{C}$, with a 30 -second dwell time at each temperature. The transfer time was 20 seconds. The entire thermal cycling protocol corresponds to 1 year of clinical condition. To facilitate specimen handling, the roots were fixed in standardized self-cured cylindrical acrylic blocks using a specially designed attachment which was attached to the dental surveyor (Ramses LTC, Egypt). The metal attachment allows positioning the long axis of the tooth parallel to metal rod. A push-out test was performed to evaluate the dowel-intraradicular dentin interfacial bond strength. The specimens were fixed to the arm of a lowspeed diamond saw and were sectioned. The direction of the sectioning was perpendicular to the long axis of the attached root and was done under water cooling. From each specimen, two dowel/dentin sections were obtained, each was $5 \mathrm{~mm}$ thick. The thickness of each slice was measured using a digital caliper and then each slice was anchored to loading fixture and fixed with cyanoacrylate glue. For performing micro push-out test, a specially designed attachment was fabricated, and it consisted of three parts, upper part which is carrying $1.0-\mathrm{mm}$-diameter cylindrical plunger that pushes the dowel segment; the plunger tip size was selected and positioned to touch only the dowel, without stressing the surrounding dowel space walls; lower part in which there is a large cavity that receives the pushed dowel fragment; and a movable part that can be placed above or removed from the large cavity of the lower part, the upper end of this movable part has a small cavity in which the root section is placed, and at the bottom of this cavity, there is a hole with a $2 \mathrm{~mm}$ diameter which is slightly larger than the dowel space diameter. Compressive loading was applied to each sample after mounting in loading fixture. A computer-controlled testing machine (Model LRX-Plus; Lloyd Instruments Ltd., Fareham, UK) with a load cell of $5 \mathrm{kN}$ (Fig. 1) was used. The data were recorded using computer software (Nexygen-MT; Lloyd Instruments Ltd.) and loaded at a crosshead speed of $0.5 \mathrm{~mm} /$ minute. Load applied by plunger $(1 \mathrm{~mm})$ diameter to ensure the plunger was positioned in contact with the dowel only to be displaced downward.

The maximum failure load was recorded in Newton and converted into megapascal. The bond strength was calculated from the recorded peak load divided by the computed surface area, as calculated by the following formula: Bond $=F / A$

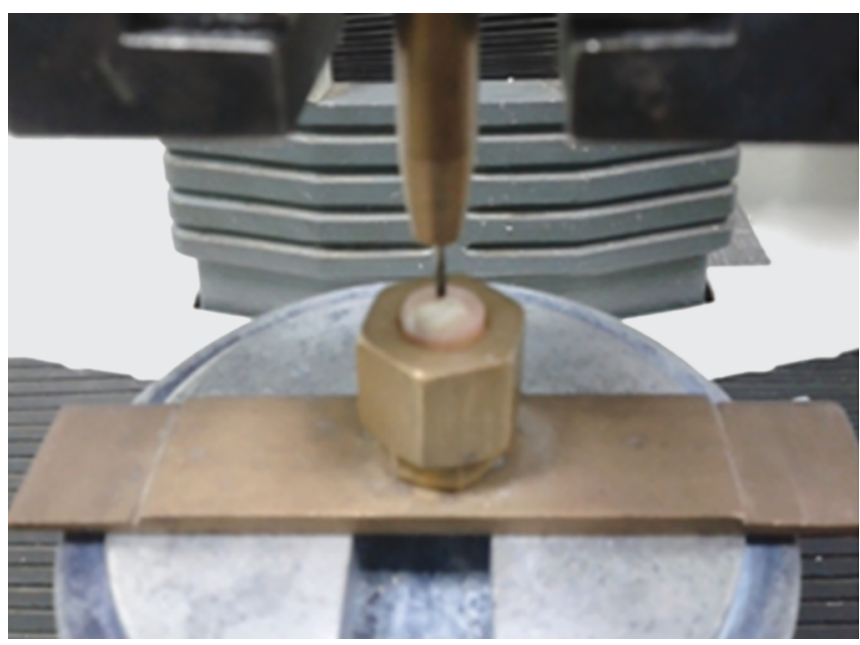

Fig. 1: Sample mounted on testing machine

$$
[A=(\pi h(r 1+r 2)],
$$

where $\pi$ is the constant $3.14, r 1$ is the apical radius, $r 2$ is the coronal one, and $h$ is the thickness of the sample in millimeters.

Failure was defined as extrusion of filling material and confirmed by sudden drop along load-deflection curve recorded by computer software (Nexygen, Lloyd Instruments Ltd.).

The statistical analysis was performed using the statistical package for social science (SPSS for Window, version 20; IBM Corp., Armonk, NY, USA). Mean values for each group were calculated, and differences between the groups were tested for statistical significance by use of two-way ANOVA and post hoc Tukey's test at $\alpha=0.05$.

\section{Results}

The two-way ANOVA procedure (Table 1) revealed a significant difference for the experimental groups, root canal regions, and the adhesion protocol $(p<0.001)$. Moreover, their interaction was also significantly different $(p<0.001)$.

Tables 2 and 3 represent the means and standard deviations (SDs) of push-out bond strength values in megapascal for all test and control groups as a function of cement types used. In control, the highest mean push-out bond strength for sound root canals $(14.8 \pm 2.1 \mathrm{MPa})$ was recorded using self-etch adhesion protocol. The lowest mean push-out bond strength value $(9.9 \pm 1.8 \mathrm{MPa})$ was recorded using total-etch adhesion protocol. In test, the highest mean push-out bond strength for perforated root canals $(8.9 \pm 1.6$ $\mathrm{MPa}$ ) was recorded for $2 \mathrm{~mm}$ perforation size apically using selfetch adhesion protocol. The lowest mean push-out bond strength value $(2.3 \pm 0.5 \mathrm{MPa})$ was recorded for $4 \mathrm{~mm}$ apical root perforation

Table 1: Two-way analysis of variance procedure

\begin{tabular}{lcclc}
\hline Source of variation & $d f$ & $M v$ & Fvalue & $p$ value \\
\hline Groups & 1 & 300.9 & 50.9 & $<0.001$ \\
Adhesion protocol & 1 & 243.2 & 30.3 & $<0.001$ \\
Perforation & 2 & 448.2 & 75.9 & $<0.001$ \\
Groups $\times$ adhesion protocol $\times$ & 9 & 534.7 & 27.4 & $<0.001$
\end{tabular}

perforation 
Perforation Size Effects on Dowel Adhesion Strength

Table 2: Means and standard deviations of push-out bond strength values (in $\mathrm{MPa}$ ) for sound root group as a function of cement used

\begin{tabular}{llcl}
\hline Root condition & Cement types & Mean value MPa & p value \\
\hline Sound & Total etch & $9.9^{\mathrm{A}}{ }_{\mathrm{a}} \pm 1.8$ & $<0.001$ \\
& Self-etch & $14.8^{\mathrm{A}}{ }_{\mathrm{a}} \pm 2.1$ & $<0.001$ \\
\hline
\end{tabular}

Different superscript letters in the same row indicates significance between regions $(p<0.001)$

Table 3: Means and standard deviations of push-out bond strength values (in MPa) for perforated root group as a function of cement used

\begin{tabular}{|c|c|c|c|c|}
\hline $\begin{array}{l}\text { Root } \\
\text { condition }\end{array}$ & Cement types & $\begin{array}{l}\text { Mean } \\
\text { value MPS of } \\
\text { perforated } \\
2 \mathrm{~mm}\end{array}$ & $\begin{array}{l}\text { Mean } \\
\text { value MPS of } \\
\text { perforated } \\
4 \mathrm{~mm}\end{array}$ & $p$ value \\
\hline \multirow[t]{2}{*}{ Perforated } & Total etch & $7.1_{b}^{A} \pm 1.1$ & $2.3_{b}^{B} \pm 0.5$ & $<0.001$ \\
\hline & Self-etch & $8.9^{A} \mathrm{~b} \pm 1.6$ & $5.9^{B}{ }_{a} \pm 1.3$ & $<0.001$ \\
\hline
\end{tabular}

Different superscript letters in the same column indicate significance between groups $(p<0.001)$

Table 4: Comparison of total push-out bond strength mean values as a function of perforation

\begin{tabular}{llll}
\hline Variable & & Mean $\pm S D$ & $p$ \\
\hline $\begin{array}{l}\text { Root canal } \\
\text { condition }\end{array}$ & Sound & $9.2 \pm 1.4$ & $<0.001$ \\
& Perforated & $6.1 \pm 1.4$ & \\
\hline
\end{tabular}

Table 5: Comparison of total push-out bond strength mean values as a function of adhesion protocol

\begin{tabular}{llll}
\hline Variable & Mean $\pm S D$ & $p$ \\
\hline $\begin{array}{l}\text { Adhesion } \\
\text { protocol }\end{array}$ & Total etch & $5.2 \pm 2.1$ & $<0.001$ \\
& Self-etch & $9.7 \pm 1.3$ & \\
\hline
\end{tabular}

using total-etch adhesion protocol. For sound roots, Tukey's post hoc test revealed a significant difference between sound root canal using total-etch and self-etch adhesion protocol $(p<0.001)$. Also, for perforated roots, Tukey's post hoc test revealed a significant difference between self-etch compared with total etch regardless of perforation size $(p<0.001)$.

Regardless of adhesion protocol or perforation size, sound root canal group recorded statistically significant difference $(p<0.001)$, with higher mean push-out bond strength value $(9.2 \pm 1.4 \mathrm{MPa})$ vs perforated root canal group $(6.1 \pm 1.4 \mathrm{MPa})$ as indicated by multifactorial ANOVA followed by pair-wise Tukey's post hoc test (Table 4).

Regardless of root canal condition, self-etch group recorded a statistically significant difference $(p<0.001)$, with higher mean push-out bond strength value $(9.7 \pm 1.3 \mathrm{MPa})$ than total-etch group $(5.2 \pm 2.1 \mathrm{MPa})$ as indicated by multifactorial ANOVA followed by pair-wise Tukey's post hoc test (Table 5).

Regarding adhesion protocol, a statistically significant difference ( $p<0.001$ ) was found in mean push-out bond strength value $(10.2 \pm 3.3 \mathrm{MPa})$ with $2 \mathrm{~mm}$ perforation apically vs $4 \mathrm{~mm}$ value $(9.1 \pm 2.4)$ as indicated by multifactorial ANOVA followed by pair-wise Tukey's post hoc test (Table 6).
Table 6: Comparison of total push-out bond strength mean values as a function of root canal perforation size

\begin{tabular}{lccc}
\hline Variable & & Mean $\pm S D$ & $p$ \\
\hline $\begin{array}{l}\text { Root canal } \\
\text { perforation size }\end{array}$ & $2 \mathrm{~mm}$ & $10.2 \pm 3.3$ & $<0.001$ \\
& $4 \mathrm{~mm}$ & $9.1 \pm 2.4$ & \\
\hline
\end{tabular}

\section{Discussion}

The use of FRC resin dowels has become popular because of elastic modulus of fiber dowels more closely resembling that of dentin compared with metal dowels. ${ }^{17-19}$ This similarity may possibly result in stress reduction between the dentin and the dowel and superior retention. ${ }^{18}$ This would result in less catastrophic failure such as root fracture. ${ }^{19}$ Accordingly, fiber dowels were chosen to be used in this study. Easy post system was selected for testing because it is a popular fiber-reinforced dowel system with zircon-enriched silicon unidirectional glass fibers ( $60 \%$ volume) which is bound in an epoxy resin matrix.

Fiber dowels are passively retained within the root canals. Fiberreinforced composite dowels are prevented from dislodgment by the luting agent adherence to the dowel and to the intraradicular dentin. ${ }^{20}$ Naumann et al. ${ }^{21}$ have also suggested that they enhance the ability of the restoration to withstand functional forces. The laboratory performance of resin luting cements, along with their easy handling, has led to their widespread clinical acceptance for dowel and core cementation. RelyX ultimate adhesive resin cement was used for cementation of fiber dowels in this study.

Dual cure resin cements may minimize the problems related to the difficulty of light reaching the most apical portions of the root canal, with polymerization being activated by the chemical composition of the activators, and thus, the most cervical portions would be benefited by light sensitization. ${ }^{22,23}$ Bond strength measurements are widely used to evaluate the effectiveness of adhesive systems and to provide useful information about the adhesion quality between materials and tooth structure. In this study, the push-out test was performed using 5-mm-thick tooth slices, because this approach offers the advantage of smaller adhesive areas, which help to avoid the difficulties of microtensile specimen preparation. ${ }^{24} \mathrm{~A}$ variety of luting agents and the corresponding adhesives can be used to bond fiber dowels into root canals. These adhesives can be of the self-etch or total-etch varieties. Self-etching systems and total-etch systems sealed the root system much better than the self-adhesive cements. Etchand-rinse alcohol-based adhesives are supposed to be applied to moist dentin, but this is almost impossible to control in the canal. Cements that contain the self-etch 10-methacryloyloxydecyl dihydrogen phosphate (10-MDP; Clearfil ${ }^{\oplus}$ Esthetic Cement; Kuraray America) react with the hydroxyapatite in the hybrid layer to form a low-soluble MDP-calcium salt to improve long-term stability. ${ }^{25}$

In this study, Clearfil SE Bond self-etching primer produced bond strength significantly higher than those of the total-etch systems on conventionally treated subgroups. Recent investigations $s^{26,27}$ evaluating Clearfil SE Bond and various adhesive systems reached similar results. It has been advocated that despite forming thinner hybrid layers $(0.5-1.0 \mu \mathrm{m})$ than those formed by the total-etch systems $(2.0-5.0 \mu \mathrm{m})$, the self-etching primers may provide bond strengths to dentin comparable or even superior to those obtained with adhesive systems that advise the acid etching as a separate step of the bonding protocol. ${ }^{27,28}$ Thus, Clearfil SE Bond can be 
considered a good root-end filling material, and the combination of etchant and primer into one system is advantageous in that it reduces the application time and technique-related sensitivity. ${ }^{28}$ This was in agreement with similar studies by Korasli et al. ${ }^{29}$ and $\mathrm{Er}$ et al. ${ }^{30}$ However, this was in disagreement with Ozturk and Özer ${ }^{31}$ and Bitter et al. ${ }^{32}$ who stated that the self-etch adhesive systems are composed of weaker acids within the primer agent compared with phosphoric acid and, therefore, less efficient in dissolving the thick smear layer observed after the preparation with drills.

Adper single bond is a total-etch adhesive to be used in combination with phosphoric acid used in this study. The use of simplified adhesive systems that combine primer and bond into one application (two-step etch-and-rinse and one-step self-etching) promotes adverse compatibility, which may compromise the bond between dentin and dual-cure resin cements. Although several perforation cases have been reported, there are limited numbers of studies published on the use of MTA in perforation repair in the cervical region of tooth that needs restoration with fiber dowels. Tooth perforations have been defined as artificial and involuntary lesions consequently communicating the pulp cavity with the periodontal ligament with prognosis depending on the location, size, shape, presence or absence of infection, and the tooth involved (anterior or posterior) so that the bacterial infection is either prevented or treated after sealing. ${ }^{33}$ Lee et al. ${ }^{15}$ described for the very first time the use of MTA in the sealing of root perforations. Since then, other authors ${ }^{34,35}$ have demonstrated the viability of this material to seal the communications between the pulp cavity and the external surface of the teeth, both in the crown and root. Osteogenesis and cementogenesis was shown to be induced by MTA. ${ }^{36}$ In this study, MTA was used in sealing root perforation due to its aforementioned characteristic and good sealing ability and high degree of biocompatibility. ${ }^{37}$

The luting of fiberglass dowels was carried out 24 hours after MTA insertion. In this study, the root perforation sealed with MTA was located at the apical region of test groups I and II, did not avoid the decreasing $(p<0.05)$ of the bond strength values of groups I and II in relation to the groups without perforations (groups III and IV). Moreover, either the patient's age or even the pulp condition can alter the dentinal substrate due to the dentinal sclerosis, which reduces the dentinal permeability and the quality of the substrate available for adhesion. ${ }^{38}$ Previous reports have shown that apical root perforation has affected the push-out bond strength. ${ }^{39}$ This study reported highest bond strength recorded in the control group irrespective of luting agent used. However, the apically perforated roots have recorded the lowest value. The result can be attributed to the inability of the cement to flow properly at the apical region and the presence of MTA which lacks adhesion property to the luting agent. On the contrary, some studies recorded higher push-out value relative to this study, which might be explained by different dentinal tubules density and diameter. ${ }^{40,41}$ Apical perforation repair with MTA was reported to achieve a favorable endodontic and periodontal prognosis; however the effect of the size of the perforation on the bond strength of the luting agent is not well documented. ${ }^{42}$ This study has shown that perforation size has an effect on the bond strength of the luting agents used if the repair was done with MTA.

Many factors should be considered to restore the esthetics and function of the teeth when adhesive procedures are required because of the clinical condition and variability of the materials available for restoration. For this purpose, new materials and further studies are necessary to assess the bond strength of the materials to both mixed substrates and teeth with perforations sealed with different materials, considering the variability of techniques and restorative materials available.

\section{Conclusion}

Few conclusions can be drawn from this study:

- Bond strength of glass fiber dowels affected by root canal condition.

- Regardless of adhesion protocol, sound root canal recorded higher mean value than perforated root canal.

- Self-etch adhesion protocol recorded higher mean value than total-etch adhesion protocol.

\section{References}

1. Goracci C, Corciolani G, Vichi A, et al. Light-transmitting ability of marketed fiber posts. J Dent Res 2008;87(12):1122-1126. DOI: 10.1177/154405910808701208.

2. Robbins JW. Restoration of the endodontically treated tooth. Dent Clin North Am 2002;46(2):367-384. DOI: 10.1016/s0011-8532(01)00006-4.

3. Wadhwani KK, Jindal R, Srivastava S, et al. Comparative Evaluation of fracture resistance of various post systems-an in vitro study. J Conserv Dent 2003;6(2):56.

4. Fernandes AS, Shetty $S$, Coutinho I. Factors determining post selection: a literature review. J Prosthet Dent 2003;90(6):556-562. DOI: 10.1016/j.prosdent.2003.09.006.

5. Sadek FT, Monticelli F, Goracci C, et al. Bond strength performance of different resin composites used as core materials around fiber posts. Dent Mater 2007;23(1):95-99. DOI: 10.1016/j.dental.2005.12.005.

6. Saupe WA, Gluskin AH, Radke JrRA. A comparative study of fracture resistance between morphologic dowel and cores and a resin-reinforced dowel system in the intraradicular restoration of structurally compromised roots. Quintessence Int 1996;27(7): 483-491.

7. Aksornmuang J, Foxton RM, Nakajima M, et al. Microtensile bond strength of a dual-cure resin core material to glass and quartz fiber posts. J Dent 2004;32(6):443-450. DOI: 10.1016/j.jdent.2004.03.001.

8. Cheung W. A review of the management of endodontically treated teeth: post, core and the final restoration. J Am Dent Assoc 2005;136(5):611-619. DOI: 10.14219/jada.archive.2005.0232.

9. Pest LB, Cavalli G, Bertani P, et al. Adhesive post-endodontic restorations with fiber posts: push-out tests and SEM observations. Dent Mater 2002;18(8):596-602. DOI: 10.1016/S0109-5641(02)00003-9.

10. Brunzel S, Yang B, Wolfart $S$, et al. Tensile bond strength of a so-called self-adhesive luting resin cement to dentin. J Adhes Dent 2010;12(2):143. DOI: 10.3290/j.jad.a17525.

11. Usumez A, Cobankara FK, Ozturk N, et al. Microleakage of endodontically treated teeth with different dowel systems. J Prosthet Dent 2004;92(2):163-169. DOI: 10.1016/j.prosdent.2004.05.004.

12. Peutzfeldt A, Sahafi A, Asmussen E. A survey of failed post-retained restorations. Clin Oral Investig 2008;12(1):37. DOI: 10.1007/s00784007-0137-9.

13. Torbjörner A, Karlsson S, Ödman PA. Survival rate and failure characteristics for two post designs. J Prosthet Dent 1995;73(5): 439-444. DOI: 10.1016/s0022-3913(05)80072-1.

14. Silveira $C M$, Sánchez-Ayala $A$, Lagravère $M O$, et al. Repair of furcal perforation with mineral trioxide aggregate: long-term follow-up of 2 cases. J Can Dent Assoc (Tor) 2008;74(8):729-733.

15. Lee SJ, Monsef $M$, Torabinejad M. Sealing ability of a mineral trioxide aggregate for repair of lateral root perforations. J Endod 1993;19(11):541-544. DOI: 10.1016/S0099-2399(06)81282-3.

16. Tsesis I, Fuss ZV. Diagnosis and treatment of accidental root perforations. Endod Topics 2006;13(1):95-107. DOI: 10.1111/j.16011546.2006.00213.x. 
17. Perdigao J, Gomes G, Augusto V. The effect of dowel space on the bond strengths of fiber posts. J Prosthodont 2007;16(3):154-164. DOI: 10.1111/j.1532-849X.2006.00166.x.

18. Bitter K, Meyer-Lückel H, Priehn K, et al. Bond strengths of resin cements to fiber-reinforced composite posts. Am J Dent 2006;19(3):138-142.

19. Qing H, Zhu Z, Chao Y, et al. In vitro evaluation of the fracture resistance of anterior endodontically treated teeth restored with glass fiber and zircon posts. J Prosthet Dent 2007;97(2):93-98. DOI: 10.1016/j.prosdent.2006.12.008.

20. Goracci C, Grandini S, Bossù M, et al. Laboratory assessment of the retentive potential of adhesive posts: a review. J Dent 2007;35(11): 827-835. DOI: 10.1016/j.jdent.2007.07.009.

21. Naumann M,Sterzenbach G, RosentrittM, et al. Is adhesive cementation of endodontic posts necessary? J Endod 2008;34(8):1006-1010. DOI: 10.1016/j.joen.2008.05.010.

22. Bitter K, Kielbassa AM. Post-endodontic restorations with adhesively luted fiber-reinforced composite post systems: a review. Am J Dent 2007;20(6):353

23. Galhano GA, De Melo RM, Barbosa SH, et al. Evaluation of light transmission through translucent and opaque posts. Oper Dent 2008;33(3):321-324. DOI: 10.2341/07-93

24. Bouillaguet $S$, Schütt $A$, Alander $P$, et al. Hydrothermal and mechanical stresses degrade fiber-matrix interfacial bond strength in dental fiber-reinforced composites. J Biomed Mater Res B Appl Biomater 2006;76(1):98-105.

25. Zicari F, Couthino E, De Munck J, et al. Bonding effectiveness and sealing ability of fiber-post bonding. Dent Mater 2008;24(7):967-977. DOI: 10.1016/j.dental.2007.11.011.

26. Pashley DH, Carvalho RM. Dentine permeability and dentine adhesion. J Dent 1997;25(5):355-372. DOI: 10.1016/S0300-5712(96)00057-7.

27. Kaaden C, Powers JM, Friedl KH, et al. Bond strength of self-etching adhesives to dental hard tissues. Clin Oral Investig 2002;6(3):155-160. DOI: 10.1007/s00784-002-0170-7.

28. Tay FR, Pashley DH. Aggressiveness of contemporary self-etching systems: I: depth of penetration beyond dentin smear layers. Dent Mater 2001;17(4):296-308. DOI: 10.1016/S0109-5641(00)00087-7.

29. Korasli D, Ziraman F, Ozyurt P, et al. Microleakage of self-etch primer/adhesives in endodontically treated teeth. J Am Dent Assoc 2007;138(5):634-640. DOI: 10.14219/jada.archive.2007.0235.

30. Er K, Taşdemir T, Bayramoğlu G, et al. Comparison of the sealing of different dentin bonding adhesives in root-end cavities: a bacterial leakage study. Oral Surg Oral Med Oral Pathol Oral Radiol Endod 2008;106(1):152-158. DOI: 10.1016/j.tripleo.2008.02.023.

31. Ozturk B, Özer F. Effect of $\mathrm{NaOCl}$ on bond strengths of bonding agents to pulp chamber lateral walls. J Endod 2004;30(5):362-365. DOI: 10.1097/00004770-200405000-00013.

32. Bitter K, Paris S, Martus $\mathrm{P}$, et al. A confocal laser scanning microscope investigation of different dental adhesives bonded to root canal dentine. Int Endod J 2004;37(12):840-848. DOI: 10.1111/j.13652591.2004.00888.x.

33. Fuss Z, Trope M. Root perforations: Classification and treatment choices based on prognostic factors. Dent Traumatol 1996;12(6): 255-264. DOI: 10.1111/j.1600-9657.1996.tb00524.x.

34. Parirokh M, Torabinejad M. Mineral trioxide aggregate: a comprehensive literature review-part III: clinical applications, drawbacks, and mechanism of action. J Endod 2010;36(3):400-413. DOI: 10.1016/j.joen.2009.09.009.

35. Roberts HW, Toth JM, Berzins DW, et al. Mineral trioxide aggregate material uses in endodontic treatment: a review of the literature. Dent Mater 2008;24(2):149-164. DOI: 10.1016/j.dental.2007. 04.007.

36. Koh ET, McDonald F, Ford TR, et al. Cellular response to mineral trioxide aggregate. J Endod 1998;24(8):543-547. DOI: 10.1016/S00992399(98)80074-5.

37. Torabinejad M, Watson TF, Ford TP. Sealing ability of a mineral trioxide aggregate when used as a root end filling material. J Endod 1993;19(12):591-595. DOI: 10.1016/S0099-2399(06)80271-2.

38. Paqué F, Luder HU, Sener B, et al. Tubular sclerosis rather than the smear layer impedes dye penetration into the dentine of endodontically instrumented root canals. Int Endod J 2006;39(1): 18-25. DOI: 10.1111/j.1365-2591.2005.01042.x.

39. Bolhuis $P$, Feilzer $A$. The influence of fatigue loading on the quality of the cement layer and retention strength of carbon fiber post-resin composite core restorations. Oper Dent 2005;30(2):220-227.

40. Bitter K, Meyer-Lueckel H, Priehn K, et al. Effects of luting agent and thermocycling on bond strengths to root canal dentine. Int Endod J 2006;39(10):809-818. DOI: 10.1111/j.1365-2591.2006.01155.x.

41. Ferrari M, Mannocci F, Vichi A, et al. Bonding to root canal: structural characteristics of the substrate. Am J Dent 2000;13(5):255-260.

42. Tsesis I, Rosen E, Tamse A, et al. Diagnosis of vertical root fractures in endodontically treated teeth based on clinical and radiographic indices: a systematic review. J Endod 2010;36(9):1455-1458. DOI: 10.1016/j.joen.2010.05.003. 